\title{
The Longitudinal Record: Linking Hepatitis A Outbreak Cases and Syndromic HL7 Data
}

\author{
Jeffrey Johnson, Lauren Kearney, Robyn Matthews, Jennifer Nelson, \\ Marjorie Richardson, Brit Colanter*, Eric McDonald and Karen Waters-Montijo
}

County of San Diego, San Diego, CA, USA

\section{Objective}

To describe how the County of San Diego linked information from a communicable disease registry and syndromic surveillance system to further describe cases associated with a large hepatitis A outbreak. Specifically, to detail the linkage process which resulted in a longitudinal understanding of individuals' hospital visits before, during, and after the reported hepatitis A incident.

\section{Introduction}

With increasing availability of syndromic meaningful use data, new approaches to disease surveillance utilizing linkages to other data systems are possible. Expanded communicable disease information may be valuable during outbreaks or other public health emergencies.

San Diego County is experiencing a significant and protracted hepatitis A outbreak. The disease has been transmitted person-toperson through close contact or through a fecally-contaminated environment, and has been primarily affecting homeless people and injection and non-injection illicit drug users. As of August 31, 2017, there were nearly 400 cases with 15 deaths. Approximately, $70 \%$ of the cases were hospitalized. This is one of the nation's largest hepatitis A outbreaks since the introduction of the hepatitis A vaccine in 1995. Additional cases are expected over the next twelve months.

The population affected by this outbreak presents some challenges for outbreak response. It is often a difficult population to reach. In addition, many have multiple comorbidities and often have health care seeking behaviors that differ from the general population. Using the medical record number (MRN) to link hepatitis A disease cases from the communicable disease registry to syndromic HL7 messages for emergency department visits and hospitalizations enabled the identification of additional hospital encounters the cases may have had before, during, or following their hepatitis A disease incident. This allowed an exploration of the ways in which this unique population interacted with the health care system in the context of a communicable disease outbreak.

This presentation will highlight the steps to link information across surveillance systems, the results, the challenges, and the benefits of linked information to public health departments.

\section{Methods}

Electronic information from a communicable disease registry system and syndromic surveillance HL7 data from participating hospitals were utilized. The patient's MRN, available in both systems, was used to link the records. The syndromic data for this project included syndromic messages from 90 days prior to the first outbreak-related hepatitis A case in November 2016 through August 31, 2017. Records with no MRN present, were unmatchable, or records with an encrypted MRN were excluded. The communicable disease registry data included outbreak-related hepatitis A cases from November 2016 through August 2017. Records were excluded if the disease incident was associated with a hospital not currently providing syndromic surveillance information. The linked dataset will continue to be updated as the outbreak progresses.

Using the linked data, relevant dates and date ranges were determined for each case, including onset of hepatitis A-associated illness, hepatitis A exposure windows, infectious periods, and a 90 day post-illness period allowing for identification of possible relapsing illness patterns. Based on these dates, hepatitis A case-patients who had HL7 messages for emergency department or hospitalization visits prior to, during, and following their hepatitis A episode were identified. Interactions with the health care system were summarized and case studies were developed.

\section{Results}

During the study time period, 396 outbreak-related hepatitis A case reports were received and documented in the communicable disease registry and nearly 18 million syndromic HL7 messages were received. After the exclusions, the MRN from 130 hepatitis A cases were linked to one or more syndromic HL7 messages associated with visits to an emergency department or inpatient hospital admissions. A total of 616 hospital encounters were documented among the 130 linked cases which reflects an overall average of 4.7 visits per case. Many of these case-patients had numerous health care visits before, during, and after their hepatitis A episode. Among the 130 linked cases, $56 \%(n=73)$ of the cases linked to one or more hospital visits other than the visit in which they were diagnosed with hepatitis A. Many of these visits were made during their infectious period prior to being treated for hepatitis A. In addition, with the available data to date, $25 \%(\mathrm{n}=33)$ of the linked cases had additional hospital visits following their hepatitis A diagnosis. These and other findings were used to provide additional outbreak response recommendations and shape additional surveillance and case monitoring approaches.

\section{Conclusions}

The use of MRN to link records from a communicable disease registry to syndromic HL7 data is a viable tool for public health departments looking to obtain additional information about communicable disease cases and enhance surveillance and disease control activities. In this study, the linkage yielded a more complete profile of patient outcomes and health care-seeking behaviors of individuals diagnosed with hepatitis A. The County of San Diego gained a broader understanding of a unique population's interactions with the health care system, including the identification of missed opportunities for vaccination and earlier diagnosis. The information was then leveraged to improve vaccination and other outreach and prevention efforts.

\section{Keywords}

Linkages; Informatics; integration; longitudinal record; meaningful use

\section{${ }^{*}$ Brit Colanter}

E-mail: brit.colanter@sdcounty.ca.gov 